\title{
Effect of microwave radiation on optical characteristics of thin gold films
}

\author{
P.M. Boltovets \\ V. Lashkaryov Institute of Semiconductor Physics, NAS of Ukraine \\ 41, prospect Nauky, 03028 Kyiv, Ukraine \\ Phone: (380-44) 525-56-26; fax: (380-44) 525-83-42; e-mail: pboltovec@mail.ru
}

\begin{abstract}
The possibility to use a complex of chemical and physical factors to form sensor surface with tunable properties was considered. It was demonstrated that the simultaneous treatment of thin gold films with guanidine thiocyanate and microwave radiation results in changes in the width of the surface plasmon resonance curve, its amplitude and position of its minimum. Thereby, the combination of these two factors can allow obtaining surface structures with controllable parameters.
\end{abstract}

Keywords: thin gold films, microwave radiation, surface plasmon resonance.

Manuscript received 24.11.10; accepted for publication 16.03.11; published online 30.06.11.

\section{Introduction}

One of the topical problems in up to date materials science is development of new approaches to creation of complex surface architectures with controllable properties for further applications in sensor systems. With this aim, different methods to form complex structures at the surface can be usually used, namely: chemical [1], biological [2], physical [3] phenomena and processes.

In particular, microwave radiation along with gamma-radiation and ultrasound processing is extensively used for modification of surfaces of different complex materials in microelectronic technologies [4]. However, its influence on properties of thin gold films widely used in sensor devices (especially in devices based on the surface plasmon resonance effect) has not been studied sufficiently yet.

The aim of this work was to investigate the influence of microwave radiation on the optical properties of thin gold films and their possible restructurization. Besides, chemically active small molecules, e.g. thiocyanate (NCS), can cause some modification of a near-surface layer in the gold film during interaction with it [5]. In particular, whereas sulfur atoms bind the surface due to their capacity to bind three atoms of Au at once, $\mathrm{CN}$ group can form a complex compound with the gold atom [6] by tearing it off the surface.
Therefore, it seemed reasonable to investigate the possibility of the mutual effect of the both factors (chemical treatment of the surface and microwave irradiation) on such characteristics of the gold film as the width of the SPR curve, its amplitude and position of the SPR minimum for the purpose to develop sensor surfaces with tunable properties.

\section{Experimental}

Glass plates (refractive index $n=1.61$ ) with an assputtered (through an adhesive Cr interlayer 1-1.5 nm thick) gold layer $50 \mathrm{~nm}$ thick [7] were treated in series with ethanol, 'piranha' (mixture of $30 \% \mathrm{H}_{2} \mathrm{O}_{2}$ and $98 \%$ $\mathrm{H}_{2} \mathrm{SO}_{4}$ in the ratio of 1:3), water, ethanol, isopropyl alcohol to remove organic contaminations.

To irradiate the samples, a magnetron with $v=2.45 \mathrm{GHz}, P=7.5 \mathrm{~W} / \mathrm{cm}^{2}$ was used. Short series of sample irradiations for 2-3 s were carried out for 10 and $20 \mathrm{~s}$. Solution of guanidine thiocyanate (GNCS) was prepared immediately before the experiment.

The following approaches to surface treatment were compared:
(a) irradiation in air;
(b) irradiation in water;
(c) irradiation in $0.01 \mathrm{M}$ GNCS;
(d) irradiation in $0.1 \mathrm{M}$ GNCS;
(e) irradiation in $1 \mathrm{M}$ GNCS. 


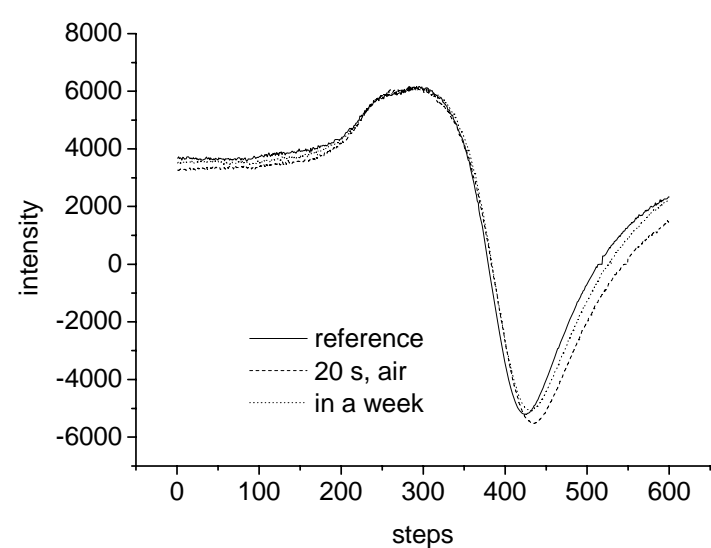

$a$

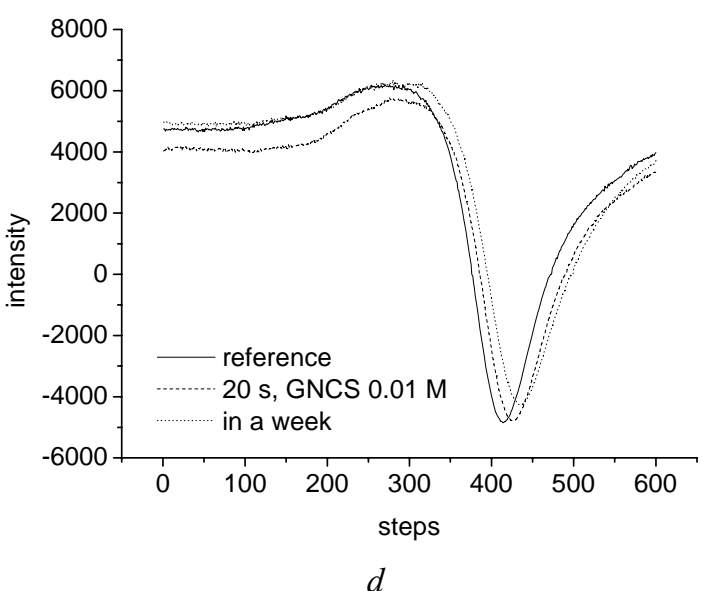

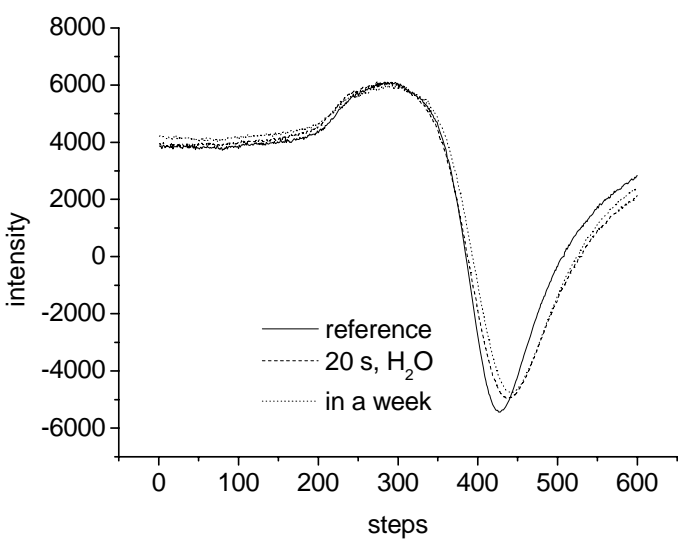

$b$

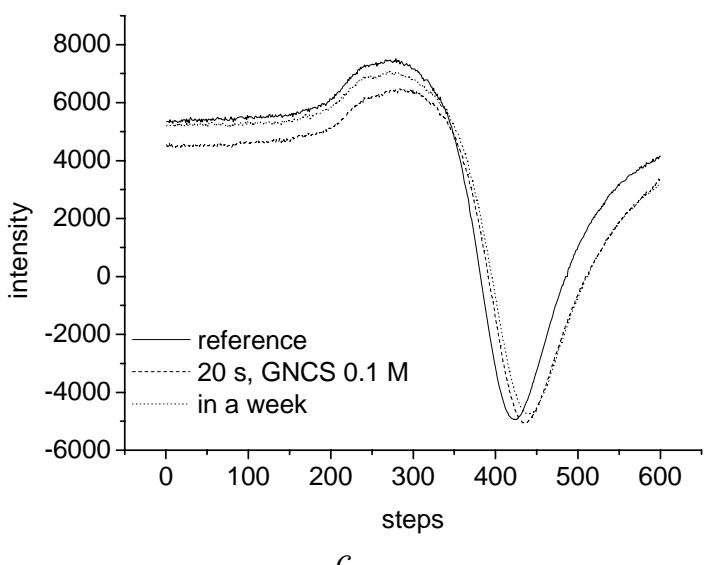

C

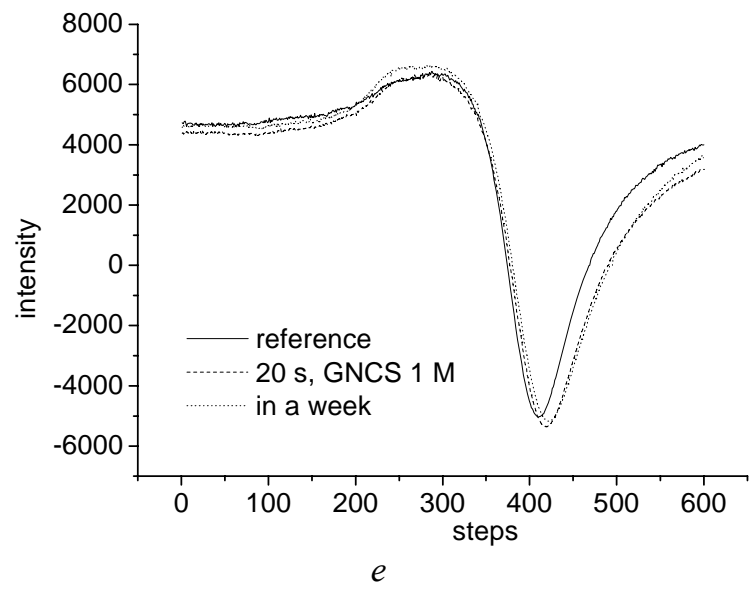

Fig. 1. The influence of microwave irradiation on the shape and position of the SPR curve: (a) irradiation in air, (b) irradiation in water, (c) irradiation in 0.01 M GNCS, (d) irradiation in 0.1 M GNCS, (e) irradiation in 1 M GNCS. 

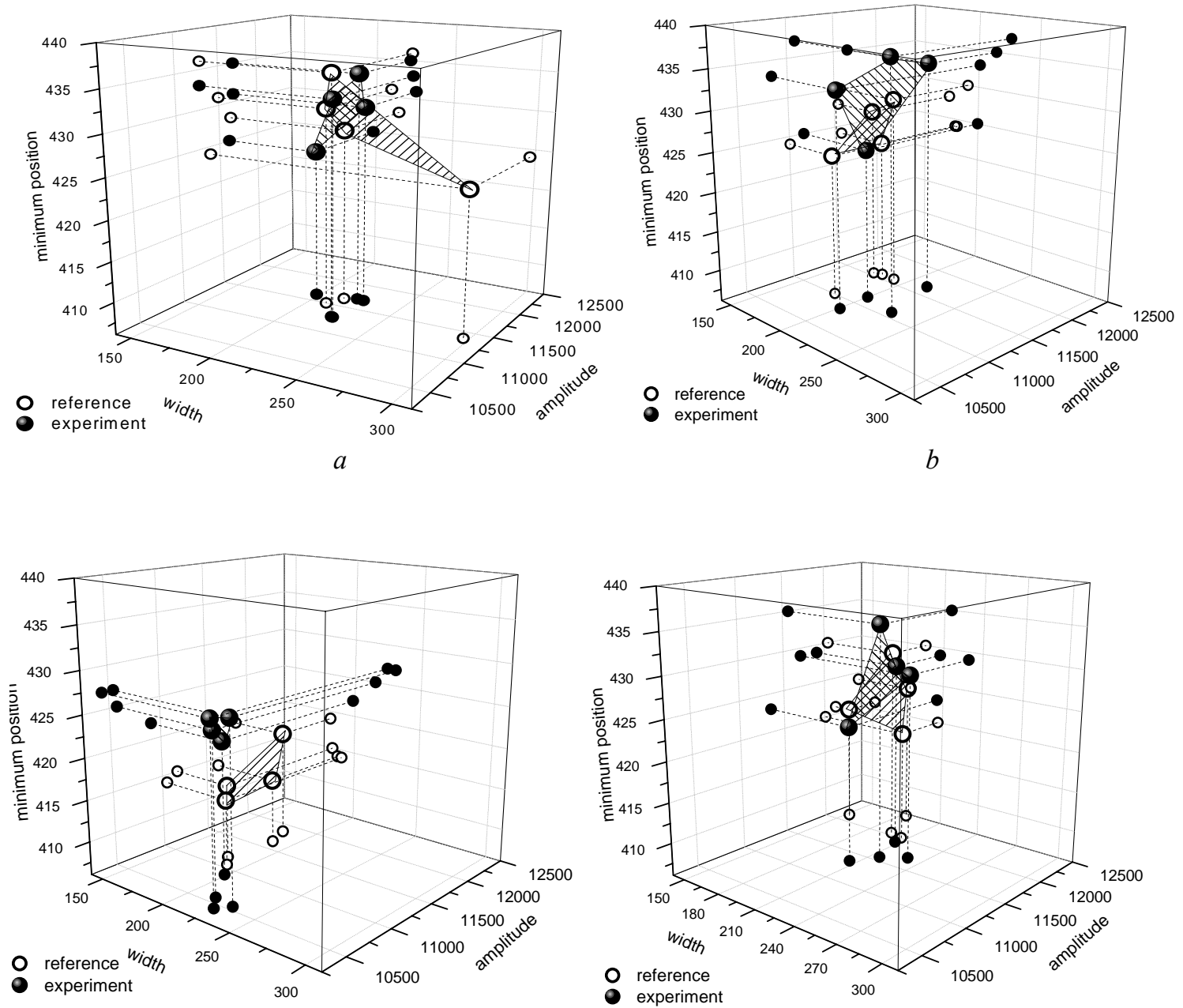

$d$

$c$

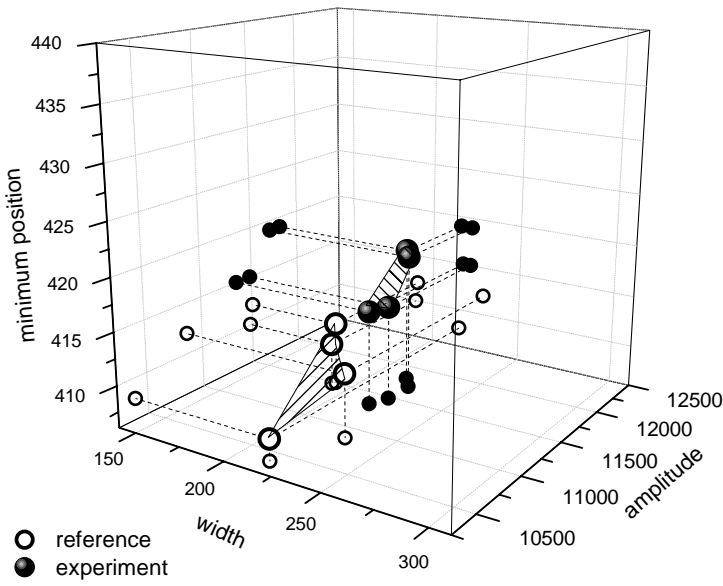

Fig. 2. Distribution of the data concerning the width of the curve, its amplitude and minimum position for different types of the surface treatment: (a) irradiation in air, (b) irradiation in water, (c) irradiation in $0.01 \mathrm{M}$ GNCS, (d) irradiation in $0.1 \mathrm{M}$ GNCS, (e) irradiation in 1 M GNCS. 
SPR spectrometer "BioHelper-01" with an open measurement architecture was used for investigations of changes in optical characteristics of thin gold films owing to modification of the surface [8]. The instrument is based on a mechanically scanning semiconductor laser $(650 \mathrm{~nm})$ with an angular resolution of $0.005^{\circ}$ and a fixed cylindrical glass prism. Single scans took $0.4 \mathrm{~s}$ and covered $23^{\circ}$.

Glass plates were fixed on a supporting glass prism (refractive index $n=1.61$ ). Optical contact was provided using an immersion liquid (polyphenyl ether, refractive index $n=1.6$ ). Four measurements were performed at each sample. Measurements were repeated in a week after the irradiation.

\section{Results and discussion}

Short series of sample irradiations for 2-3 s carried out for $10 \mathrm{~s}$ did not allow to obtain statistically reliable results, though minor variation in the position of the SPR minimum were observed for samples treated by microwave radiation as compared with untreated ones (data are not shown). In the case of the short series of sample irradiations for $20 \mathrm{~s}$, a well-defined tendency to the shift of the SPR minimum position to the increase of the SPR angle as well as diminution of the amplitude and slight broadening the SPR curve (Fig. 1).

However, no direct correlation between the GNCS concentration and the level of manifestation of the observed effect was found. Also, there was no significant difference between the results of measurements carried out in air and in the solution. Measurements that were carried out in a week demonstrated that except the case of irradiation in air there was no surface relaxation, and the minimum of the reflected light remained shifted relative to its position in reference measurements.

More detailed analysis of the curve parameters shows that in the case of irradiation in air there is no well-defined clusterization of the indications before and after irradiation for such parameters as the width of the curve and minimum position. However, some tendency to more clear discrimination of the data before and after irradiation is visible for the amplitude (Fig. 2a).

However, in water medium the distribution of the data concerning the width of the curve and position of the minimum is better, whereas the change in the amplitude after irradiation is less pronounced (Fig. 2b). A positive tendency to clusterization by all three parameters is manifested for the case of irradiation of the samples in the GNCS $0.01 \mathrm{M}$ solution (Fig. 2c) as well as in the $0.1 \mathrm{M}$ one (Fig. 2d). However, increase in the GNCS concentration results in the decline of the clusterization level (Fig. 2e). It is most visible for the width and amplitude of the curve.
In general, it is possible to state that concerning the width of the SPR curve, its amplitude and minimum position the values of these parameters form welldefined clusters for treated and untreated sensor surface except cases of irradiation in air and in high concentration of GNCS. Besides, the clear regularity concerning the broadening of the SPR curve, decrease in its amplitude and increase of the SPR angle is observed. It can serve as an evidence of the decrease in the thickness of the films under consideration as a consequence of chemical treatment as well as possible formation of nanostructured architectures under microwave radiation. Thereby, the combination of these two factors can allow obtaining surface structures with controllable parameters.

\section{References}

1. P.M. Boltovets, S.A. Kravchenko, B.A. Snopok, Building interfacial nanostructures by sizecontrolled chemical etching // Plasmonics, 5(4), p. 395-403 (2010).

2. E. Brynda, M. Houska, A. Brandenburg, A. Wikerstal, Optical sensors for real-time measurement of analytes in blood plasma // Biosensors and Bioelectronics, 17, p. 665 (2002).

3. S.A. Zynio, A.V. Samoylov, E.R. Surovtseva, Y.M. Shirshov, V.M. Mirsky // Sensors, 2, p. 62-70 (2002).

4. A.B. Kamalov, Modification of Au-Ti (W, Cr, $\mathrm{TiB}_{\mathrm{x}}$ )-GaAs contacts properties caused by external influences // Radioelectron. Communs. Systems, 52 (3), p. 160-164 (2009).

5. P.M. Boltovets, N.S. Dyachenko, B.A. Snopok, Yu.M. Shirshov, Ya.D. Lampeka, Proteinorientating structures: thiocyanate layers as support for immobilization of bioreceptors // Proc. SPIE, 4425, p. 189-193 (2001).

6. L. Polling, P. Polling, Chemistry. Moscow, Mir, 1978 (in Russian).

7. G.V. Beketov, Yu.M. Shirshov, O.V. Shynkarenko, V.I. Chegel, Surface plasmon resonance spectroscopy: prospects of superstate refractive index variation for separate extraction of molecular layer parameters // Sensors and Actuators B, 48, p. $432-438$ (1998).

8. B. Snopok, M. Yurchenko, L. Szekely, G. Klein, E. Kasuba, SPR based immuno-capture approach for in vitro analysis of protein complex formation: mapping of MRS18-2 binding site on retinoblastoma protein // Anal. Bioanal. Chem. 386, p. 2063-2073 (2006). 